\title{
Hematological Parameters and Its Utility in Dengue Fever: A Prospective Study
}

\author{
Kinjal Patel $^{1^{*}}$, Dipanki Patel ${ }^{2}$, V. K. Das ${ }^{3}$ \\ ${ }^{1}$ Pathologist, Department of Pathology, Shri Vinoba Bhave Civil Hospital, Silvassa, Dadra and Nagar Haveli, India \\ ${ }^{2}$ Head of the Department, Department of Pathology, Shri Vinoba Bhave Civil Hospital, Silvassa, Dadra and Nagar Haveli, India \\ ${ }^{3}$ Director, Medical and Public Health Services, Shri Vinoba Bhave Civil Hospital, Dadra and Nagar Haveli, India
}

\begin{abstract}
Background: Dengue is a major preventable and treatable cause of morbidity and mortality among children and adults that occurs mainly in tropical and subtropical regions. Early diagnosis of dengue is important for provision of special care which ensures marked reduction in the morbidity. Hematological examination is an essential step in the management of these patients. Hence this study was undertaken to document the hematological changes in dengue fever. Aims and Objectives: To co-relate the hematological data during the evolution of dengue fever and to predict the severity of the disease. Methods: The prospective study of 260 cases of positive dengue cases and its hematological profile was conducted over a period of four months between July 2014 to October 2014 at Department of Pathology, Shri Vinoba Bhave Civil Hospital, Silvassa. Results: In this study, total 260 dengue NS1 antigen ELISA positive patients were analyzed. The most common hematological abnormality was thrombocytopenia (68.46\%) followed by leucopenia (56.92\%), atypical lymphocytes (52.30\%) and lymphocytosis (49.23\%). Conclusion: Awareness, early recognition \& early diagnosis are important for favorable outcome. Hematological parameters are very helpful for disease monitoring and can be helpful in prediction of prognosis. Early and prompt diagnosis of symptom with complete hemogram and dengue test can save a life.
\end{abstract}

Keywords: Dengue fever, Hematological profile, Thrombocytopenia, Leucopenia, Lymphocytosis.

\section{Introduction}

Dengue is caused by one of the four serotypes of dengue virus ( DEN-1, DEN-2, DEN-3 AND DEN-4 ) also referred to as an arbovirus ( arthropod - borne viruses ) that belongs to genus Flavivirus of the family Flaviviridae. ${ }^{1,}$ It is a disease with a wide clinical spectrum and wide variety of presentations, ranging from asymptomatic to an undifferentiated fever ( viral syndrome) to the more severe forms such as severe dengue (SD) or Dengue hemorrhagic fever (DHF). ${ }^{1}$

Transmission to humans occurs by the bite of the female Aedes aegypti mosquito infected by one of four serotypes of the virus. This mosquito, a domestic species adapted to urban conditions, is the main vector in India. ${ }^{2}$

The period of transmission from humans to mosquitoes begins one day before the start of fever up to the next day of illness corresponding to the viremia phase. After a female bites an individual in the viremia phase, viral replication (extrinsic incubation) begins in the vector in from eight to twelve days. In humans, the incubation period ranges from 3 to 15 days (intrinsic incubation) with an average of 5 days. ${ }^{3}$

According to estimates of the World Health Organization (WHO), about 50 million cases of dengue fever occur annually worldwide and 2.5 billion people live in risk areas. In 2005, the International Health Regulation (IHR), included dengue fever as an emergent public health disease, with implications for health safety due to the spread of the epidemic beyond national boundaries. ${ }^{4}$

\section{Material and Methods}

This was a prospective study of 260 patients with dengue infection at Shri Vinoba Bhave Civil Hospital during the period from July 2014 to October 2014. The case definition of dengue haemorrhagic fever was based on clinical history according to WHO criteria ${ }^{3}$, characterised by acute high grade fever for 2-7 days, haemorrhagic manifestations e.g epistaxis and gum bleeding, platelet count less than 100x $10^{9} / \mathrm{L}$ and haemoconcentration ( rising packed cell volume $>$ $20 \%$ of baseline ). Dengue shock syndrome was defined as presentation of symptoms identical to dengue fever and dengue haemorrhagic fever and complicated by clammy skin, frigid limbs, restlessness, weak pulse which is virtually indiscernible and narrow pulse pressure (less than 20 $\mathrm{mmHg}$ ).

All showed positive dengue NS1 serology performed by NS1 capture enzyme -linked immunosorbant assay (ELISA) manufactured by Panbio diagnostics. The standard protocol of collection of serum and storing at $-20{ }^{0} \mathrm{C}$ was adopted. All the patients irrespective of age and gender were included in the study.

Complete hemogram including hemoglobin, hematocrit, total count, differential count and platelet count were noted of 260 patients.

Hemogram profile was done on Automated Hematology analyzer Sysmex KX- 21.

Peripheral smear examination was done in all the patients. 


\section{International Journal of Science and Research (IJSR) ISSN (Online): 2319-7064 \\ Index Copernicus Value (2013): 6.14 | Impact Factor (2015): 6.391}

\section{Results}

In this study, total 260 dengue NS1 antigen ELISA positive patients were included and analyzed.

The patients were both 176 (67\%) males and 84 (33\%) females ranging from 1 year- 76 years.

Table 1: Haemoglobin

\begin{tabular}{|c|c|c|}
\hline Haemoglobin $(\mathrm{gm} / \mathrm{dl})$ & Results & Percentage \\
\hline$<10.0$ & $32 / 260$ & $12.30 \%$ \\
\hline $10-15$ & $200 / 260$ & $76.93 \%$ \\
\hline$>15$ & $28 / 260$ & $10.77 \%$ \\
\hline
\end{tabular}

Table 2: Haematocrit

\begin{tabular}{|c|c|c|}
\hline Haematocrit (\%) & Results & Percentage \\
\hline$<35$ & $64 / 260$ & 24.61 \\
\hline $35-45$ & $166 / 260$ & 63.85 \\
\hline$>45$ & $30 / 260$ & 11.54 \\
\hline
\end{tabular}

Table 3: Total leucocyte count

\begin{tabular}{|c|c|c|}
\hline $\begin{array}{c}\text { Total leucocyte count } \\
\text { ( Cells/cumm) }\end{array}$ & Results & Percentage \\
\hline$<4,000$ & $148 / 260$ & 56.92 \\
\hline $4,000-11,000$ & $108 / 260$ & 41.54 \\
\hline$>11,000$ & $04 / 260$ & 01.54 \\
\hline
\end{tabular}

Table 4: Percentage of Lymphocytes

\begin{tabular}{|l|l|l|}
\hline Lymphocytes & Results & Percentage \\
\hline Lymphocytes $>45 \%$ & $128 / 260$ & 49.23 \\
\hline Atypical lymphocytes & $136 / 260$ & 52.30 \\
\hline
\end{tabular}

Table 5: Platelet profile

\begin{tabular}{|c|c|c|}
\hline $\begin{array}{c}\text { Platelet count } \\
\text { ( per cumm) }\end{array}$ & Results & Percentage \\
\hline$<20,000$ & $09 / 260$ & 03.46 \\
\hline $21000-50,000$ & $72 / 260$ & 27.70 \\
\hline $51,000-1,00,000$ & $97 / 260$ & 37.30 \\
\hline $1,00,000-1,50,000$ & $64 / 260$ & 24.62 \\
\hline$>1,50,000$ & $18 / 260$ & 06.92 \\
\hline
\end{tabular}

Laboratory investigations revealed that the most common haematological abnormality was thrombocytopenia in 178 (68.46\%) patients (platelet count less than 1,00,000/ cumm) followed by leucopenia ( total leucocyte count $<4,000$ ) in 148 (56.92 \%) patients. However, in 72 (27.70\%) patients platelet count was below 50,000/cumm and only 09 (03.46\%) cases had platelet count below 20,000/cumm at the time of presentation. Atypical lymphocytosis was reported in 136 (52.30\%) cases. Leucopenia with lymphocytosis were seen in $42(16.15 \%)$ patients. Other hematological parameters are shown in Table I-V.

\section{Outcome}

There was one mortality due to the disease. Patient was NS1 positive with dengue hemorrhagic fever. The patient had severe thrombocytopenia, hemoconcentration and raised hematocrit.

\section{Discussion}

Due to changing climate, urbanization, poor living conditions and inadequate waste management, vector born diseases like dengue fever are becoming more common. Although vector control programs are launched in endemic countries every year, yet dengue fever has become a serious problem worldwide. India being a tropical country provides suitable weather for aedes mosquito to grow and an increase in the disease burden has been noticed in recent years. ${ }^{5}$

In our study, there was a slight male preponderance. Similar observations have been made by Malathesha et $\mathrm{al}^{6}$ and Banerjee et al. ${ }^{2}$ Hypothetically, this can be related to the increased risk of exposure of male gender to mosquito bites due to occupational and recreational activities.

The most significant laboratory abnormality seen in our patients was thrombocytopenia, as also observed by Malathesa et $\mathrm{al}^{6}$ and $\mathrm{N}$ Ali et al. ${ }^{7}$ Thrombocytopenia is due to the direct and antibody mediated destruction of the platelets and megakaryocytes and also due to the suppression of the bone marrow by virus. ${ }^{8}$

Haemorrhagic manifestation are very common with severe thrombocytopenia and severity of haemorrhagic tendency correlates with the platelet counts. Platelet count was evaluated by Sysmex KX- 21 as well as on peripheral smear. The counter gives a false low reading when large platelets are present. Such cases were abviated by assessment of platelets on smear.

Leucopenia was observed in $56.92 \%$ of patients as also observed by Malathesha et $\mathrm{al}^{6}$ and Banerjee et al. ${ }^{2}$ Leucopenia is due to the direct marrow suppression by the virus. $^{8}$

We observed an increase in concentration of atypical lymphocytes. Similar findings have been reported by N Ali et $\mathrm{al}^{7}$ and it mainly represents that serum immunoglobulin production is enhanced during dengue viral infection, these are mostly against the specific serotype and obviously not protective to the infections caused by other serotypes. ${ }^{9}$

Infection with dengue fever offers lifelong immunity but the cross immunity is minimal and short lived. The main complication of the dengue fever is the development of dengue hemorrhagic fever (DHF). Pathogenesis of DHF depends both on the host factors and viral factors. It is observed in the literature DHF risk increases in secondary infection. Various mechanisms have been described to explain occurrence of DHF in secondary infection which includes $\mathrm{T}$ - cell mediated immunoreactions, secondary antibody dependant immunoreactions and compliment activation by antigen and antibody reaction. Studies have shown elevated level of cytokines, interferon y, interleukin-2 and a chain of CD8, CD4 and interleukin-2. All the complement levels are decreased except C3, however these mechanisms still needs to be fully defined. ${ }^{10}$

Dengue fever is a self limiting disease. Dengue hemorrhagic fever causes morbidity and mortality. No antiviral treatment is available hence fluid and electrolyte replacement and supportive therapy are the available modalities of treatment. Since no vaccine for this disease, vector control is the only way to check the transmission of disease. ${ }^{11}$ 


\section{Conclusion}

Most, if not all, deaths from Dengue are preventable with appropriate and timely medical attention. The hemogram is the most important guide to therapy and prognosis. Most of the cases in our study had thrombocytopenia, leucopenia and lymphocytosis with atypical forms. These indicators, if rightly and timely assessed can be of value for better care of complicated cases.

\section{References}

[1] Chambers TJ, Hahn CS, Galler R, Rice CM. F lavivirus genome organization, expression and replication. Annu Rev Microbio 1990;44:649-88.

[2] Banerjee M, Chateerjee T, Choudary GS, Srinivas V, Kataria VK. Dengue: A Clinico - Haematological Profile. Medical Journal Armed Forces India. 2008; 64 (4): 333-6.

[3] World Health Organization. Dengue : Guidelines for diagnosis, treatment, prevention and control Geneva: WHO: 2009.

[4] Schatzmayr HG. Viroses emergentes. Cad Saude Publica.2001; 17(suppl): 209-13.

[5] Munde DD, Shetkar UB, Clinical Features And Hematological Profile of Dengue Fever, Indian Journal Of Applied Research; vol 3 Issue 1, Jan 2013, 131-132.

[6] M K Malthesa, Ashwini H N. Hematological Manifestations in Dengue Fever- An Observational Study. Journal of Evolution of Medical and Dental Sciences 2014; Vol.3,Issue 09, March 3; Page: 22452250.

[7] N Ali, M Usman, N Syed, M Khurshid. Haemorrhagic manifestations and utility of Haematological parameters in dengue fever. Scandinavian Journal of Infectious Disease, 2007; 39: 1025-1028.

[8] Jameel T, Mehmood K, Mujaba G, Choudhry N, Afzal $\mathrm{N}$, Paul RF. Changing haematological parameters in dengue viral infections. J Ayub Med Coll Abbottabad. 2012 Jan- Mar; 24(1): 3- 6.

[9] Wilder- Smith A, Schwartz E. Dengue in Travelers. N Engl J M 2005;353: 924-32.

[10] Kalayanarooj S, Vaughn DW, Nimmannitya S, Green S, Suntayakorn S, Kunentrasai N, etal. Early clinical and laboratory indicators of acute dengue illness. $\mathrm{J}$ Infect Dis.1997 Aug; 176(2): 313-21.

[11] Guzman MG, Kouri G, Dengue: an Update. THE LANCET Infectious Diseases 2002; 2: 33- 41. 